\title{
A NOVEL ANALYTICAL METHOD TO DETECT ADULTERATION OF VIRGIN OLIVE OIL BY OTHER OILS
}

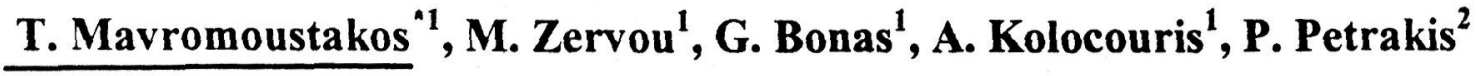

${ }^{\mathrm{I}}$ National Hellenic Research Foundation, Institute of Organic and Pharmaceutical Chemistry, 48 Vas. Constantinou Ave, 11635 Athens, Greece, tel:+301-7273869, email:tmavro@eie.gr.

${ }^{2}$ Ministry of Agriculture \& Forestry, Div. Of Informatics, Natural Rsource Monitoring, Acharnon 381, 11143 Athens, Greece, tel:+301-2112336

\begin{abstract}
In our previous publication (T. Mavromoustakos et al. J. Magn. Reson 35, S3-7, 1997) it has been shown that ${ }^{13} \mathrm{C}-\mathrm{NMR}$ spectroscopy is a valuable technique to quantitatively analyze the most abundant fatty acid moieties of the triacylglycerols of virgin olive oil. The present study focuses on the olefinic region of virgin olive oil ${ }^{13} \mathrm{C}$-NMR spectrum which shows twelve peaks resonating between 127.5-130 ppm. These peaks are assigned to the most abundant unsaturated fatty acid moieties oleic and linoleic acids of the olive oil, present in $\alpha$ and $\beta$ positions of the glycerol backbone. With the use of an internal reference pyrazine, these peaks were integrated and their areas were expressed in $\mathrm{mmol} / \mathrm{g}$ of virgin olive oil. The intensities of the twelve observed peaks are affected when an authentic virgin olive oil is mixcd with a secd-oil. This observation is used to develop a method to detect adulteration based on ${ }^{13} \mathrm{C}$ NMR spectroscopy. The novel method is superior to the applied classical ones that involve wet chemistry because it is faster and not destructive.
\end{abstract}

